Transactions for 1862. The fundamental theorem is a relation $a-(b-\kappa)+\left(c-\kappa^{\prime}+\pi\right)-\left(d-\kappa^{\prime \prime}+\pi^{\prime}-\omega\right)=0$ existing in any figure whatever between $a$ the number of points, $b$ the number of lines, $c$ the number of areas, $d$ the number of spaces, and certain supplementary quantities $\kappa, \kappa^{\prime}, \kappa^{\prime \prime}, \pi, \pi^{\prime}, \omega$. In an exteusive class of figures these last are each $=0$, and the relation is $a-b+c-d=0$; thus, in a closed box, $a=8, b=12, c=6, d=2$ (viz., there is the finite space inside, and the infinite space outside, the box): if the box be opened, $a=10, b=15$, $c=6, d=1$; if the lid be taken away, $a=8, b=12, c=5, d=1$; in each case, $a-b+c-d=0$. If the bottom be also taken away, $a=8, b=12$, $c=4, d=1$; bat here one of the supplementary quantities comes in, $x^{\prime \prime}=1$, and the theorem is $a-b+c-\left(d-x^{\prime \prime}\right)=0$. The chief difficulty and interest of the Memoir consist in the determination of the supplementary quantities $\kappa, \kappa^{\prime}, \kappa^{\prime \prime}, \pi, \pi^{\prime}, \omega$.

Dr. Henrici then read the following paper :-

"On certain formula concerning the Theory of Discriminants; with applications to Discriminants of Discriminants, and to the Theory of Polar Curves." By Olados M. F. E. Henrici, Ph.D.

Let

$$
u=\left(a^{\prime}, a^{\prime \prime}, a^{\prime \prime \prime}, \ldots \gamma\left(y_{1}, y_{2}, y_{3} \ldots\right)^{m}\right.
$$

be a quantic in the variables $y_{1}, y_{2}, y_{3}, \ldots$ and let be its discriminant. is a function of the coefficients $a^{\prime}, a^{\prime \prime}, a^{\prime \prime \prime} \ldots$ If these coefficients are related by the equation $=0$, then $u=0$ has a system of double roots, which I denote by $\eta_{1}, \eta_{2}, \eta_{3} \ldots$. For these values of and of the variables $y$, the proportion holds

$$
\frac{\delta u}{\delta a^{\prime}}: \frac{\delta u}{\delta a^{\prime \prime}}: \frac{\delta u}{\delta a^{\prime \prime \prime}}: \ldots=\stackrel{\delta a^{\prime \prime}}{\delta a^{\prime}}: \frac{\delta \Xi^{\prime}}{\delta a^{\prime \prime}}: \frac{\delta a^{\prime \prime}}{\delta a^{\prime \prime \prime}}: \ldots,
$$

which is used to determine the singular roots $\eta$, as the $\frac{\delta u}{\delta \eta}$ are products of powers of the $\eta$ 's. If the coefficients $a$ in $u$, altogether' or some of them, be any functions whatsoever of one or more new variables

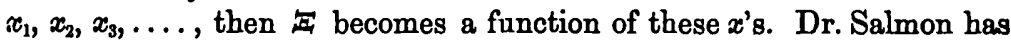
proved, in the second edition of his "Lessons introductory to the modern higher Algebra," that the above proportion is true also for the differential coefficients of with respect to these $x$ 's. Or we have

$$
\frac{\delta u}{\delta x_{1}}: \frac{\delta u}{\delta x_{2}}: \frac{\delta u}{\delta x_{3}}: \ldots \ldots=\frac{\delta \Xi_{1}}{\delta x_{1}}: \frac{\delta \Xi}{\delta x_{2}}: \frac{\delta \Xi}{\delta x_{3}}: \ldots \ldots
$$

The only condition that both proportions may hold is that should vanish, and that the variables $y$ should be replaced by the singular roots $\eta$. Hence both proportions hold simultaneously, and we may combine 
them into one. Denoting by $a$ any one of the coefficients $a^{\prime}, a^{\prime \prime}, a^{\prime \prime \prime}, \ldots$, and by $x$ any one of the variables $x_{1}, x_{2} \ldots$, we obtain thas

$$
\frac{\delta u}{\delta a}: \frac{\delta u}{\delta x}=\frac{\delta[}{\delta a}: \frac{\delta \underline{I}}{\delta x}
$$

or, as I prefer to write it,

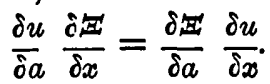

The proportion (2) is in fact a simple consequence of the first. contains $x$ implicitly only in the $a^{\prime}$ 's ; hence we have for the partial differential coefficient of $z$ with respect to $x$,

$$
\frac{\delta \Xi}{\delta x}=\frac{\delta a^{\prime}}{\delta a^{\prime}} \frac{\delta a^{\prime}}{\delta x}+\frac{\delta \Xi}{\delta a^{\prime \prime}} \frac{\delta a^{\prime \prime}}{\delta x}+\frac{\delta g^{\prime}}{\delta a^{\prime \prime \prime}} \frac{\delta a^{\prime \prime \prime}}{\delta x}+\ldots ;
$$

and by aid of (1),

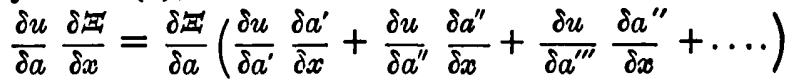

$$
\begin{aligned}
& =\frac{\delta g}{\delta a} \frac{\delta u}{\delta a},
\end{aligned}
$$

which is (3). It was in this way that I first arrived at the formula (3), and it is not without interest to see (3) proved by (1), as it shows that (3) holds for $a n y$ function of the coefficients $a^{\prime}, a^{\prime \prime}, a^{\prime \prime \prime} \ldots$, for which the proportion (1) holds. This is the case, for instance, with the first differentials $\frac{\delta \Xi^{\prime}}{\delta a}$ as soon as $\eta_{1}, \eta_{2}, \eta_{3} \ldots$ represent not merely a system of double roots but of treble roots of $u=0$.

The formula (3) is very useful in a great number of problems, which depend upon discriminants involving one or more variables. The factor $\frac{\delta u}{\delta a}$ on the left-hand side is a product of powers of the singular roots $\eta_{1}, \eta_{2}, \eta_{3}, \ldots$ and therefore in general does not vanish. We can at least always find one coefficient $a$ for which $\frac{\delta u}{\delta a}$ is not $=0$, as all the variables do not vanish simultaneously; or we may transform our quantio $u$ by linear transformation in such a way, that none of the singular roots vanish. I shall therefore always suppose $\frac{\delta u}{\delta a}$ not to be $=0$. Then (3) shows that $\frac{\delta E}{\delta x}$ vanishes in two cases, either if $\frac{\delta(E x}{\delta a}=0$, or if $\frac{\delta u}{\delta x}=0$. To see whether in these cases $\frac{\delta^{2}-\underline{z}}{\delta x^{2}}$ also vanishes, it is necessary to develope some new formulø. For shortness' sake I take a binary quantic, and suppose the coefficients functions of one variable $x$ only, the whole proceeding being easily transferred to quantics in general. 
Let $f(y, 1)$, or more shortly $f(y)$, be a binary quantic

$$
f(y)=a_{m} y^{m}+a_{m-1} y^{m-1}+\ldots . . .+a_{1} y+a_{0},
$$

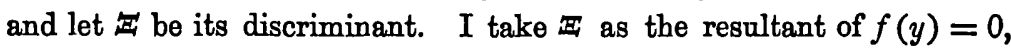
$f^{\prime}(y)=0$. If $y_{1}, y_{2}, \ldots y_{m-1}$ be the roots of $f^{\prime}(y)=0$, we have

$$
\text { 业 }=a_{m}^{m-2} f\left(y_{1}\right) f\left(y_{2}\right) \ldots f\left(y_{m-1}\right) \text {. }
$$

The coefficients $a_{k}$ are supposed functions of $x$. Then the $y_{i}^{\prime}$ s become functions of $x$ determined by the equation $f^{\prime}(y)=0$. From this expression of $\approx$ we obtain

$$
\mathbb{F}^{\prime}(x)=a_{m}^{m-2} \frac{d f\left(y_{1}\right)}{d x} f\left(y_{2}\right) f\left(y_{3}\right) \ldots+\ldots
$$

where all terms not written vanish with $f\left(y_{1}\right)=0$. If now $x=\xi$ be a root of $=0, y=\eta$ the corresponding double root of $f(y)=0$ and $y_{1}=\eta$, hence $f\left(y_{1}\right)=f(\eta)=0$, we have

$$
\mathbb{F}^{\prime}(\xi)=a_{m}^{m-2} \frac{d f(\eta)}{d \xi} f\left(y_{2}\right) f\left(y_{3}\right) \ldots \ldots
$$

Now

$$
\frac{d f(\eta)}{d \xi}=\frac{\delta f(\eta)}{\delta \xi}+\frac{\delta f(\eta)}{\delta \eta} \frac{\delta \eta}{\delta \xi}=\frac{\delta f(\eta)}{\delta \xi},
$$

as $\frac{\delta f(\eta)}{\delta \eta}=0$. We may therefore, in the expression for $\xi^{\prime}(\xi)$, replace the total differential coefficient $\frac{d f(\eta)}{d \xi}$ by the partial one $\frac{\delta f(\eta)}{\delta \xi}$.

To find a simple expression for the factor $a_{m}^{m-2} f\left(y_{2}\right) f\left(y_{3}\right) \ldots$ of $\xi^{\prime}(\xi)$, I suppose $x$ remains unaltered and equals $\xi$. Then the coefficients $a_{\xi}$ have constant values, which satisfy the equation $\xi=0$. From these $I$ separate one, say $a_{k}$, and substitute $a_{k}+\sigma$ for $a_{k}$. this value of $\sigma$ corresponds again the double root $y=\eta$ of $f(y)$. Differentiating with regard to $\sigma$, we obtain, exactly as before, for $x$

$$
\frac{\delta \xi_{1}^{\prime}}{\delta \sigma}=a_{m}^{m-2} \frac{\delta f(\eta)}{\delta \sigma} f\left(y_{2}\right) f\left(y_{3}\right) \ldots,
$$

as soon as $\sigma=0$. Bat $\frac{\delta f(\eta)}{\delta \sigma}=\frac{\delta f(\eta)}{\delta a_{k}}=\eta^{k}$, and $\frac{\delta \xi \xi}{\delta \sigma}=\frac{\delta \xi}{\delta a_{k}}$, whence

$$
\mathbb{E}_{k}=a_{m}^{m-2} \frac{\delta f(\eta)}{\delta a_{k}} f\left(y_{2}\right) f\left(y_{3}\right) \ldots
$$

where $z_{k}$ is written for $\frac{\delta}{\delta a_{k}}$. This in combination with the above expression for $\mathbb{E}^{\prime}(\xi)$ gives

or

$$
\begin{aligned}
\frac{\delta f(\eta)}{\delta a_{k}} \boldsymbol{\Xi}^{\prime}(\xi) & =\mathbb{z}_{k} \frac{\delta f(\eta)}{\delta \xi}, \\
\eta^{k} \mathbb{\Xi}^{\prime}(\xi) & =\Xi_{k} \frac{\delta f(\eta)}{\delta \xi} .
\end{aligned}
$$

As we suppose that $\frac{\delta f(\eta)}{\delta a_{k}}\left(=\eta^{k}\right)$ does not vanish, this formula shows 
that $\xi^{\prime}(\xi)$ vanishes, or that $\xi=0$ has a double root $x=\xi$, when either $\xi_{k}=0$ or $\frac{\delta f(\eta)}{\delta \xi}=0$, and in no other case. These cases are to be separated.

1. Let $\xi_{k}=0, \frac{\delta f(\eta)}{\delta \xi}$ not $=0$. When this is satisfied for one index $k$, it must be satisfied for every $k$, or else we must have $\frac{\delta f(\eta)}{\delta a_{k}}=\eta^{k}=0$ against our supposition. The vanishing of every $z_{k}$ is the condition that $f(y)$ may have two pairs of double roots $\eta_{1}, \eta_{2}$, or one treble root $\eta$.

Differentiating twice with respect to $x$, we get

$$
\mathbb{F}^{\prime \prime}(x)=2 a_{m}^{m-2} \frac{\delta f\left(y_{1}\right)}{\delta x} \frac{\delta f\left(y_{2}\right)}{\delta x} f\left(y_{3}\right) \ldots+\ldots,
$$

where all terms not written vanish with $f\left(y_{1}\right)=0, f\left(y_{2}\right)=0$; or, if we substitute $x=\xi, y_{1}=\eta_{1}, y_{2}=\eta_{2}, f\left(y_{1}\right)=f\left(y_{2}\right)=0$, we have

$$
\xi^{\prime \prime \prime}(\xi)=2 a_{m}^{m-2} \frac{\delta f\left(\eta_{1}\right)}{\delta \xi} \frac{\delta f\left(\eta_{2}\right)}{\delta \xi} f\left(y_{3}\right) \ldots
$$

If we further substitute $a_{i}+\rho$ and $a_{k}+\sigma$ for $a_{i}$ and $a_{k}$, whilst $x$ remains $=\xi$, we find, by differentiating with respect to $\rho$ and $\sigma$, and making $\rho=\sigma=0$,

$$
\begin{aligned}
\xi_{i k}=\frac{\delta^{2} \xi^{\prime}}{\delta a_{i} \delta a_{k}} & =a_{m}^{m-2}\left(\frac{\delta f\left(\eta_{1}\right)}{\delta a_{i}} \frac{\delta f\left(\eta_{2}\right)}{\delta a_{k}}+\frac{\delta f\left(\eta_{2}\right)}{\delta a_{i}} \frac{\delta f\left(\eta_{1}\right)}{\delta a_{k}}\right) f\left(y_{3}\right) \ldots \\
& =\left(\frac{\delta f\left(\eta_{1}\right)}{\delta a_{i}} \frac{\delta f\left(\eta_{2}\right)}{\delta a_{k}}\right) a_{m}^{m-2} f\left(y_{3}\right) \ldots
\end{aligned}
$$

These two expressions for $\mathbb{E}^{\prime \prime}(\xi)$ and Fik $_{i k}$ hold simultaneously, and may be combined into

$$
\left(\frac{\delta f\left(\eta_{1}\right)}{\delta a_{i}} \frac{\delta f\left(\eta_{2}\right)}{\delta a_{k}}\right) \xi^{\prime \prime}(\xi)=2 \xi_{i k} \frac{\delta f\left(\eta_{1}\right)}{\delta \xi} \frac{\delta f\left(\eta_{2}\right)}{\delta \xi},
$$

which holds good for two distinct double roots $\eta_{1}, \eta_{2}$.

Similarly we find

$$
\frac{\delta f(\eta)}{\delta a_{i}} \frac{\delta f(\eta)}{\delta a_{k}}, \mathbb{z}^{\prime \prime}(\xi)=\xi_{i k}\left(\frac{\delta f(\eta)}{\delta \xi}\right)^{2}
$$

for a treble root. Here

$$
\begin{aligned}
z_{i k} & =\frac{\delta^{2}}{\delta a_{i} \delta a_{k}}, \\
\left(\frac{\delta f\left(\eta_{1}\right)}{\delta a_{i}} \frac{\delta f\left(\eta_{2}\right)}{\delta a_{k}}\right) & =\frac{\delta f\left(\eta_{1}\right)}{\delta a_{i}} \frac{\delta f\left(\eta_{2}\right)}{\delta a_{k}}+\frac{\delta f\left(\eta_{1}\right)}{\delta a_{k}} \frac{\delta f\left(\eta_{2}\right)}{\delta a_{i}} .
\end{aligned}
$$

When for one value $x=\xi$ a greater number of double roots $\eta_{1}, \eta_{2} \ldots \eta_{p}$ exists, we get 


$$
\left(\frac{\delta f\left(\eta_{1}\right)}{\delta a_{i_{1}}} \frac{\delta f\left(\eta_{2}\right)}{\delta a_{i_{2}}} \ldots \frac{\delta f\left(\eta_{\mu}\right)}{\delta a_{i_{\mu}}}\right) \xi^{\mu}(\xi)=1.2 \ldots \mu \Xi_{i_{1} i_{2} \ldots i_{\mu}} \frac{\delta f\left(\eta_{1}\right)}{\delta \xi} \ldots \frac{\delta f\left(\eta_{\mu}\right)}{\delta \xi},
$$

where the expression within the brackets denotes the sum of all the 1.2... $\mu$ similar expressions, which are obtained by permutating all the $\eta_{k}$ 's. When two of these double roots $\eta_{1}, \eta_{2}$ coincide, forming a treble root $\eta$, this formula changes into

$$
\left.\left(\frac{\delta f(\eta)}{\delta a_{i_{2}}} \frac{\delta f(\eta)}{\delta a_{i_{3}}} \frac{\delta f\left(\eta_{3}\right)}{\delta a_{i_{3}}} \ldots\right)\right)^{\mu}(\xi)=1.2 \ldots \mu \boldsymbol{\xi}_{i_{2} i_{3} \ldots i_{\mu}}\left(\frac{\delta f(\eta)}{\delta \xi}\right)^{2} \frac{\delta f\left(\eta_{3}\right)}{\delta \xi} \ldots
$$

and similarly when more roots coincide.

2. Let $\frac{\delta f(\eta)}{\delta \xi}=0$, but $\xi_{k}$ not $=0$. For such a double root $x=\xi$ of there exists one double root $\eta$ of $f(y)$ only. Differentiating twice, we obtain

$$
\xi^{\prime \prime}(\xi)=a_{m}^{m-2} \frac{d^{2} f(\eta)}{d \xi^{2}} f\left(y_{2}\right) f\left(y_{8}\right) \ldots
$$

Here we cannot replace the total differential coefficient $\frac{d^{2} f(\eta)}{d \xi^{2}}$ by the partial one. We have to develope $\frac{d^{2} f(\eta)}{d \xi^{2}}$, and to substitute the value of $\frac{\delta \eta}{\delta \xi}$, which is obtained by differentiating the equation $f^{\prime}(y)=0$.

The result is

$$
\frac{d^{2} f(\eta)}{d \xi^{2}}=\frac{\frac{\delta^{2} f(\eta)}{\delta \xi^{2}} \frac{\delta^{2} f(\eta)}{\delta \eta^{2}}-\left(\frac{\delta^{2} f(\eta)}{\delta \xi \delta \eta}\right)^{2}}{\frac{\delta^{2} f(\eta)}{\delta \eta^{2}}}
$$

In combination with a former equation, we thus find

$$
\begin{aligned}
\frac{\delta f(\eta)}{\delta a_{k}} \xi^{\prime \prime}(\xi) & =\xi_{k} \frac{d^{2} f(\eta)}{d \xi^{2}} \\
& =\xi_{k} \frac{\frac{\delta^{2} f(\eta)}{\delta \xi^{2}} \frac{\delta^{2} f(\eta)}{\delta \eta^{2}}-\left(\frac{\delta^{2} f(\eta)}{\delta \xi \delta \eta}\right)^{2}}{\frac{\delta^{2} f(\eta)}{\delta \eta^{2}}} .
\end{aligned}
$$

The denominator $\frac{\delta^{2} f(\eta)}{\delta \eta^{2}}$, in general, does not vanish. This would indicate a treble root $\eta$ of $f(\eta)=0$.

These different cases, in which $\xi$ can become a double root of $\xi$, are clearly distinguished by a geometrical representation of $f(y)$. If $\boldsymbol{x}$ and $y$ are taken as coordinates of a point, $f(y)=0$ represents a curve. $z=0$ gives the equation to all tangents $x=\xi$ parallel to the axis of $y$. If for one of these tangents two donble roots $\eta_{1}, \eta_{2}$ exist, it becomes a 
double tangent; and it is a tangent of inflexion in case of a treble root. In both cases the $z_{k}$ 's vanish. But if $\frac{\delta f(\eta)}{\delta \xi}=0$, the curve has a double point, which absorbs two tangents; and if besides $\frac{d^{2} f}{d \xi^{2}}=0$, this double point becomes a cusp, which absorbs three tangents, and has a treble root.

When in case of a double tangent $\frac{\delta f}{\delta \xi}=0$ for one point of contact,

this becomes a double point. We then have $\mathbf{m}^{\prime \prime}(\xi)=0$, and

$$
\left(\frac{\delta f\left(\eta_{1}\right)}{\delta a_{i}} \frac{\delta f\left(\eta_{i}\right)}{\delta a_{k}}\right) \xi^{\prime \prime \prime}(\xi)=3 \Xi_{i k} \frac{d^{2} f\left(\eta_{1}\right)}{d \xi^{2}} \frac{\delta f\left(\eta_{2}\right)}{\delta \xi} ;
$$

and so on for other cases.

All that has been said about discriminants of binary functions holds for quantics in general. Assuming the coefficients to be functions of more than one variable, our formuli hold for any one of them. Some new ones are easily added for the higher differential coefficients with respect to more variables.

It is further clear that we may substitute resultants in general for discriminants. I have treated of discriminants only, because the applications $I$ intend to make belong to discriminants alone.

\section{Discriminants of Discriminants.}

I suppose now that in our quantic

$$
f(y)=a_{m} y^{m}+a_{m-1} y^{m-1}+\ldots+a_{1} y+a_{0},
$$

the coefficients $a_{k}$ are integral functions in $x$ all of the degree $n$,

$$
\text { Hence } f(y) \text { may be written }
$$

$$
a_{k}=\alpha_{n}^{(k)} x^{n}+\alpha_{n-1}^{(k)} x^{n-1}+\ldots+a_{0}^{(k)} \text {. }
$$

$$
f(y)=u_{0}+x u_{1}+x^{2} u_{2}+\ldots+x^{n} u_{n}
$$

where the $u_{n}$ 's are binary quantics in $(y, 1)$ of the order $n$, with coefficients $\alpha_{i}^{(k)}$. The discriminant 2 is (in 2$)$ of the degree $2 n(m-1)$,

$$
\underline{E}=\mathrm{A}_{0}+\mathrm{A}_{1} x+\mathrm{A}_{2} x^{2}+\ldots
$$

I form the discriminant $D$ of 1 , and shall show that

$$
\mathrm{D}=\Delta \mathrm{B}^{2} \mathrm{C}^{3} \text {, }
$$

where $\mathrm{B}=0$, of the order $4 n(m-2)(m-3)$ in the $a_{i}^{(k)} s_{s}$, is the condition that $f(y)$ may have for a certain value of $x$ two donble roots, where $\mathrm{C}=0$, of the order $6 n(m-2)$, is the condition for a treble root of $f(y)$, and where $\Delta=0$, of the order $6 m n-4(m+n)+4$, is the condition that for a double root $\eta$ of $f(y)$ the differential coefficient $\frac{\delta f(\eta)}{\delta \xi}$ may vanish. $\Delta$ therefore is the discriminant of $f$, treated as a function of $x$ and $y$. 
This theorem is, according to Dr. Salmon, given by Prof. Cayley for $n=1$, or for $f(y)=u_{0}+x u_{1}$, but without a general proof. By aid of the formulø before developed, the proof is very easy.

$\mathrm{D}=0$ is the condition that $\Xi$ shall have a double root $\xi$. But we have seen that has a double root only in one of the three cases where $\Delta, \mathrm{B}$, or $\mathrm{C}$ vanishes. Hence $\mathrm{D}$ must contain these quantities as factors, and it cannot contain any other factor. To show that they enter as a linear, squared, or cubed factor respectively, I suppose the coefficients $a_{i}^{(k)}$ of $f(y)$ linear functions of a new variable $\lambda$. Then $\Delta, \mathrm{B}, \mathrm{C}$ become functions of $\lambda$.

Let $\lambda$ be a root of $\mathrm{B}=0$, then has a double root $\xi$, and $f(y)$ has two double roots $\eta_{1}, \eta_{2}$. For these values we have, as $\mathrm{D}$ is the discriminant of $\boldsymbol{s}$,

$$
\begin{aligned}
\frac{\delta \Xi}{\delta \mathrm{A}_{k}} \mathrm{D}^{\prime}(\lambda) & =\frac{\delta \mathrm{D}}{\delta \mathrm{A}_{k}} \frac{\delta \xi}{\delta \lambda}, \\
\text { and also } & \frac{\delta f(\eta)}{\delta a_{k}} \frac{\delta \Xi}{\delta \lambda}=\Xi_{k} \frac{\delta \Xi}{\delta \lambda} .
\end{aligned}
$$

Now for two double roots $\eta_{1}, \eta_{2}$ of $f(y)$ we have $\Psi_{k}=0$, hence $\frac{\delta}{\delta \lambda}=0$, hence also $\mathrm{D}^{\prime}(\lambda)=0$. Or each root $\lambda$ of $B$ is a double root of $\mathrm{D}$, or $\mathrm{B}^{2}$ is a factor of $\mathrm{D}$.

Further, for the same values of $\lambda, x, y$,

$$
\frac{\delta \Xi}{\delta \mathrm{A}_{k}} \mathrm{D}^{\prime \prime}(\lambda)=\frac{\delta \mathrm{D}}{\delta \mathrm{A}_{k}} \frac{d^{2} \Xi}{d \lambda^{2}} .
$$

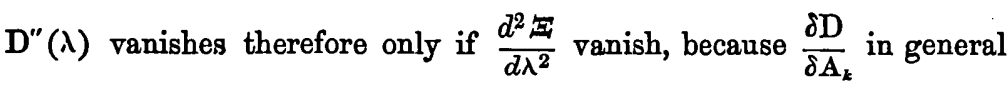
does not vanish. This would indicate that for one value of $\lambda$ two double roots $\xi$ of exist. But

$$
\frac{d^{2} \pi}{d \lambda^{2}}=\frac{\frac{\delta^{2}-}{\delta \xi^{2}} \frac{\delta^{2} \xi}{\delta \lambda^{2}}-\left(\frac{\delta^{2} \xi}{\delta \xi \delta \lambda}\right)^{2}}{\frac{\delta^{2} \xi}{\delta \xi^{2}}},
$$

or $\mathrm{D}^{\prime \prime}(\lambda)$ vanishes together with

$$
\frac{\delta^{2}-\delta^{2}}{\delta \xi^{2}} \frac{\delta^{2}}{\delta \lambda^{2}}-\left(\frac{\delta^{2}-\xi^{2}}{\delta \xi \delta \lambda}\right)^{2}
$$

For the same values of $\lambda, \xi, \eta_{1}, \eta_{3}$ we have

and similarly

$$
\left(\frac{\delta f\left(\eta_{1}\right)}{\delta a_{i}} \frac{\delta f\left(\eta_{2}\right)}{\delta a_{k}}\right) \frac{\delta^{2} \xi}{\delta \xi^{2}}=2 \Xi_{i k} \frac{\delta f\left(\eta_{1}\right)}{\delta \xi} \frac{\delta f\left(\eta_{2}\right)}{\delta \xi},
$$

$$
\left(\frac{\delta f\left(\eta_{1}\right)}{\delta a_{i}} \frac{\delta f\left(\eta_{2}\right)}{\delta a_{k}}\right) \frac{\delta^{2} z^{\prime}}{\delta \xi \delta \lambda}=\xi_{i k}\left(\frac{\delta f\left(\eta_{1}\right)}{\delta \xi} \frac{\delta f\left(\eta_{2}\right)}{\delta \lambda}\right),
$$




$$
\left(\frac{\delta f\left(\eta_{1}\right)}{\delta a_{i}} \frac{\delta f\left(\eta_{2}\right)}{\delta a_{k}}\right) \frac{\delta^{2}-\Xi^{2}}{\delta \lambda^{2}}=2 \Xi_{i k} \frac{\delta f\left(\eta_{1}\right)}{\delta \lambda} \frac{\delta f\left(\eta_{2}\right)}{\delta \lambda} .
$$

Introducing these values in

$$
\frac{\delta^{2}-2}{\delta \xi^{2}} \frac{\delta^{2}-\delta^{2}}{\delta \lambda^{2}}-\left(\frac{\delta^{2}}{\delta \lambda \delta \xi}\right)^{2}
$$

we obtain an expression which in general does not vanish. Hence B is a squared factor only of $\mathrm{D}$.

But our expression vanishes as soon as the two double roots $\eta_{1}, \eta_{2}$ coincide with a treble root. In this case, therefore, $D^{\prime \prime}(\lambda)$ vanishes. This $\lambda$ is a root of $C=0$. Hence each root of $C$ is a treble root of $D$, or $\mathrm{C}^{3}$ is a factor of $\mathrm{D}$. It is easily seen that $\mathrm{C}$ cannot be a factor of a higher degree.

The third factor $\Delta$ of $D$ enters only in the first degree. For we have

$$
\frac{\delta \mathrm{I}^{\prime}}{\delta \mathrm{A}_{k}} \mathrm{D}^{\prime}(\lambda)=\Xi^{\prime}(\lambda) \frac{\delta \mathrm{D}}{\delta \mathrm{A}_{k}}
$$

and neither $\mathbb{A}^{\prime}(\lambda)$ nor $\frac{\delta \mathrm{D}}{\delta \mathrm{A}_{k}}$ vanishes necessarily when $\Delta=0$. Therefore, as above stated, $\mathrm{D}=\Delta \cdot \mathrm{B}^{2} \cdot \mathrm{C}^{3}$.

To ascertain the orders of $\Delta, \mathrm{B}$, and $\mathrm{C}, \mathrm{I}$ first determined the order of $\mathrm{B}$ and $\mathrm{C}$ by methods indicated by Professor Cayley and Dr. Salmon, and then subtracted the order of $\mathrm{B}^{2} \mathrm{C}^{3}$ from that of $\mathrm{D}$, to find the order of $\Delta$.

The quantities $\mathrm{B}, \mathrm{C}$, and $\Delta$ are of very different character. Writing $f(y)$ in the form

$$
f(y)=u_{0}+x u_{1}+x^{2} u_{2}+\ldots+x^{n} u_{n},
$$

$\mathrm{B}$ and $\mathrm{C}$ are simultaneous invariants of the $u_{k}$ 's; but $\Delta$ is an invariant of $f$, taken as a function of $x$ and $y . \quad \Delta$ therefore remains unaltered by linear transformation of $x$ and $y$, whilst $\mathrm{B}$ and $\mathrm{C}$ are altered. But $\mathrm{B}$ and $\mathrm{C}$ retain their invariable character for certain transformations of higher order. If for $y$ such a rational function of $x$, and a new variable $y^{\prime}$, be substituted, that $y^{\prime}$ also can be expressed as a rational function of $x$ and $y$, and if for the transformed function $f$ the quantities $\mathrm{B}^{\prime}, \mathrm{C}^{\prime}$ are formed analogous to $\mathrm{B}$ and $\mathrm{C}$, then $\mathrm{B}^{\prime} \mathrm{C}^{\prime}$ must vanish together with $\mathrm{B}$ and $\mathrm{C}$ respectively; and vice versâ. This becomes evident by the geometrical representation of $f(y)$, before mentioned ; and still more so, when we make use of Riemann's peculiar representation of algebraical functions by surfaces, having as many leaves as the function has branches. Our function $f(y, x)$ is exactly of that form from which Riemann starts in his theory of Abel's functions. The substitution mentioned is of that kind which Riemann recommends for the transformation of algebraical functions into others which lead to the same class of Abel's functions. For these transformations, therefore, tho invariants $\mathrm{B}$ and $\mathrm{C}$ must be of importance. 
I add the remark, that the theorem about discriminants of discriminants is also true, if for the quantic $f(y)$ another is substituted containing any number of variables $y_{1}, y_{2}, y_{3} \ldots$, as long as the coefficients remain functions of one variable only. The proof given holds for this case. Professor Cayley has given the theorem for binary and ternary functions.*

\section{On Polar Curves.}

Let

$$
v=\Sigma a_{\kappa \lambda \mu} x_{1}^{\kappa} x_{2}^{\lambda} x_{3}^{\mu}, \quad \kappa+\lambda+\mu=r,
$$

be a ternary quantic of the order $r$, and let the coefficients $a_{\kappa \lambda \mu}$ be quantics with three other variables $y_{1}, y_{2}, y_{3}$ of the order $m$. Then $v=0$ represents as a function of the $x$ 's a system of curves of the order $r$, of such a nature that $m^{2}$ curves pass through any two given points. Or, all curves, $v=0$, which pass through one given point form a series of the index $m^{2}$. Such series of curves are formed by the $m$ th polars of all points in a plane with respect to a curve of the order $n=m+r$. The $y$ 's represent in this case the coordinates of the poles. I shall use the term pole also in the geueral case for the point of which the $y$ 's are the coordinates.

Between the curves $v=0$ will be an infinite number having a double point, but only a finite number with two double points or a cusp. The condition for a double point of $v$ is

$v_{1}=0, \quad v_{2}=0, \quad v_{3}=0$,
where $v_{i}=\frac{1}{r} \frac{\delta v}{\delta x_{i}} . \quad$ By eliminating the $x$ 's between these equations, we obtain the discriminant $\Delta$ of $v$, of the order $3 m(r-1)^{2}$ in the $y$ 's. Hence the poles $(y)$ of curves $v$ with double points form a curve $\Delta=0$ of the order $3 m(r-1)^{2}$.

If we eliminate the $y$ 's between the above equations, we obtain $a$ function $\mathrm{K}$ of the $x$ 's, the locus of the double points of the curves $v$. It is of the order $3 m^{2}(r-1)$.

The two curves $\Delta, K$ correspond point for point, to each point $(y)$ on $\Delta$ corresponds one point $(x)$ on $\mathrm{K}$, the double point of the curve $v$ for the pole $(y)$. I shall prove :-The poles $(y)$ for which $v$ has two double points are double points of $\Delta$; and the poles $(y)$ for which $v$ has a cusp are cusps of $\Delta$.

$\Delta$ is the discriminant of $v$; hence we have, for any point $(y)$ on $\Delta=0$, and for the corresponding point $(x)$ on $\mathrm{K}$,

$$
\frac{\delta v}{\delta a_{\kappa \lambda \mu}} \frac{\delta \Delta}{\delta y_{i}}=\Delta_{k \lambda \mu} \frac{\delta v}{\delta y_{i}}, \text { where } \Delta_{k \lambda \mu}=\frac{\delta \Delta}{\delta a_{\kappa \lambda \mu}} .
$$

Salmon, Geometry of Three Dimensions, 2nd edit., p. 179, foot note; and Lessons introductory to the Modern Higher Algebra, 2nd edit, p. $1+9$. 
This shows at once that $\frac{\delta \Delta}{\delta y_{i}}=0$, as soon as every $\Delta_{\kappa \lambda \mu}=0$, or that $\Delta$ has a double point as soon as $v$ has two double points; because the condition for two double points on $v$ is, that all differentials of $\Delta$ with respect to the coefficients $a_{\kappa \lambda \mu}$ vanish, or that every $\Delta_{\kappa \lambda \mu}=0$.

When every $\Delta_{k \lambda \mu}$ vanishes, we have for the second differential coefficients $\frac{\delta^{2} \Delta}{\delta y_{i} \delta y_{k}}$ the formula

$$
\left(\frac{\delta v^{\prime}}{\delta a_{\kappa \lambda \mu}} \frac{\delta v^{\prime \prime}}{\delta a_{\kappa^{\prime} \lambda^{\prime} \mu^{\prime}}}\right) \frac{\delta^{2} \Delta}{\delta y_{i} \delta y_{k}}=\Delta_{\kappa \lambda \mu, \kappa^{\prime} \lambda^{\prime} \mu^{\prime}}\left(\frac{\delta v^{\prime}}{\delta y_{i}} \frac{\delta v^{\prime \prime}}{\delta y_{k}}+\frac{\delta v^{\prime \prime}}{\delta y_{i}} \frac{\delta v^{\prime}}{\delta y_{k}}\right)
$$

where $v^{\prime}$ means $v$ for the coordinates of the one, and $v^{\prime \prime}$ for the coordinates of the other double point. In case that these two double points coincide, $v$ has a cusp. Then $v^{\prime}=v^{\prime \prime}$, and our formula changes into

$$
\frac{\delta v}{\delta a_{\kappa \lambda \mu}} \frac{\delta v}{\delta a_{\kappa^{\prime} \lambda^{\prime} \mu^{\prime}}} \frac{\delta^{2} \Delta}{\delta y_{i} \delta y_{k}}=\Delta_{\kappa \lambda \mu, \kappa^{\prime} \lambda^{\prime} \mu^{\prime}} \frac{\delta v}{\delta y_{i}} \frac{\delta v}{\delta y_{k}} .
$$

This enables us to form the Hessian of $\Delta$ for the point $(y)$, for which $v$ has a cusp. We find it proportional to

$$
\left|\begin{array}{lllll}
\frac{\delta v}{\delta y_{1}} \frac{\delta v}{\delta y_{1}}, & \frac{\delta v}{\delta y_{1}} \frac{\delta v}{\delta y_{2}^{\prime}}, \frac{\delta v}{\delta y_{1}} \frac{\delta v}{\delta y_{3}} \\
\frac{\delta v}{\delta y_{2}} \frac{\delta v}{\delta y_{1}}, & \frac{\delta v}{\delta y_{2}} \frac{\delta v}{\delta y_{2}}, \frac{\delta v}{\delta y_{2}} \frac{\delta v}{\delta y_{3}} \\
\frac{\delta v}{\delta y_{3}} \frac{\delta v}{\delta y_{1}}, & \frac{\delta v}{\delta y_{3}} \frac{\delta v}{\delta y_{2}}, \frac{\delta v}{\delta y_{3}} \frac{\delta v}{\delta y_{3}}
\end{array}\right|
$$

It vanishes identically; and so do all first minors. Hence $\Delta$ has a cusp. Q. E. D.

These properties of $\Delta$ are sufficient to determine its class. The double points and cusps of $\Delta$ are the solutions common to every $\Delta_{k \lambda \mu}=0 . \quad \Delta$ vanishes for the same values. Now, if $\Delta=0$, the coordinates of the double point of $v$ are determined by proportions, of which one is

$$
x_{1}^{r}: x_{1}^{r-1} x_{2}: \ldots \ldots: x_{2}^{r}=\Delta_{r, 0,0}: \Delta_{r-1,1,0}: \ldots \ldots: \Delta_{0, r, 0} .
$$

These show, if $\Delta_{r, 0,0}=0$, either every $\Delta_{r \lambda \mu}$ vanishes, or $x_{1}=0$. In the last case, $\Delta_{r, 0,0}$ vanishes like $x_{1}^{r}$, or the curve $\Delta_{r, 0,0}$ has here a multiple point with $r$ branches. We see the common solutionis of every $\Delta_{k \lambda \mu}$ are those solutions of $\Delta=0, \Delta_{r, 0,0}=0$ for which $x_{1}$ is not $=0$. The double points $(x)$ on $v$, for which $x_{1}=0$, are the points where the line $x_{1}=0$ meets the curve $\mathrm{K}$; their number is $3 m^{2}(r-1)$. As $\Delta_{r, 0,0}$ has here $r$ branches, the number of solutions common to $\Delta, \Delta_{r, 0,0}$, where voL. 11.-No. 16. 
$x_{1}=0$ is $3 m^{2} r(r-1)$. Subtracting this from the number of solutions of $\Delta, \Delta_{r, 0,0}$, or from $3 m^{2}(r-1)^{2}\left(3 r^{2}-6 r+2\right)$, we obtain

$$
3 m^{2}(r-1)(r-2)\left(3 r^{2}-3 r+1\right)
$$

as the number of solutions common to every $\Delta_{k \lambda \mu}=0 . \quad \Delta$ has here either a double point, in which case the curve $\Delta_{r, 0,0}$ meets $\Delta$ in two points. Or $\Delta$ has a cusp, and then the curve $\Delta_{r, 0,0}$ meets $\Delta$ in three points; because all curves $\Delta_{\kappa \lambda \mu}$ touch the tangent at a cusp of $\Delta$. If we therefore denote the number of double points by $\delta$ and the number of cusps by $k$, we have

$$
2 \delta+3 \kappa=3 m^{2}(r-1)(r-2)\left(3 r^{2}-3 r+1\right) .
$$

It follows that the curve $\Delta$ is of the class

$$
3 m(r-1)(2 m r-m-r+1) \text {. }
$$

Taking the curves $v$ as polars to a curve $u$ of the order $n=m+r$, we obtain curves $K$ and $\Delta$ for the polars of each order, except the linear ones. These curves are related in the following way:-Tho curve $\Delta$ for the $m^{\text {th }}$ polars is the curve $\mathrm{K}$ for the $(n-m-1)^{\text {th }}$ polars, and vice versâ. Steiner calls these curves "Conjugirte Kerncurven." For the first polars, or for $m=1, r=n-1$, the curve $\mathrm{K}$ is the Hessian, and $\Delta$ is that curve which Cremona calls the Steinerian. I shall denote all these curves by $\mathrm{S}$, with an index indicating for which polars they form the curve $\Delta$. Then $S^{(m)}$ and $S^{(n-m-1)}$ are conjugate, and $\mathbf{S}^{(n-2)}$ especially is the Hessian.

The curve $\mathrm{S}^{(m)}$ is the locus of the poles of $m^{\text {th }}$ polars which have double points, and it is the locus of the double points of the $(n-m-1)^{\text {th }}$ polars. The conjugate curve $\mathrm{S}^{(n-m-1)}$ is the locus of the double points of the $m^{\text {th }}$ polars, and the locus of the poles of the $(n-m-1)^{\text {th }}$ polars with double points. Their order and class follow directly from the numbers given before for $\Delta$. These numbers are, for $m=1$, given by Steiner without a proof. Cremona has determined them by geometrical, and Professor Clebsch by analytical methods. The other curves for $m$ different from 1 are only mentioned by Steiner.* Cremona and Clebsch do not treat of them. We have further the poles of $m^{\text {th }}$ polars with two double points are double points of $\mathrm{S}^{(m)}$, and the poles of the $n^{\text {th }}$ polars with a cusp are cusps of $\mathbf{S}^{(m)}$. This theorem is also known for $m=1$. Steiner gives it for the double points. Clebsch has guessed the theorem for cusps, and

* Steiner, Crollo's Journ., t. 47, p. 4. Cremona, Introd. ad ana teoria geom. dollo curve piano, Art. XX., $118(b)$. Clebsch, Crelle's Journ., t. 64, Ueber einige von Steiner behaudelto Curvon.

Stciner, speaking of tho curves $S^{(1)}$ and $S^{(n-2)}$, says :- "Dicse Curvon werdon "nobst andern' conjugirte Kerncurven genannt." These "other" curves are, no doubt, tho curves $S^{(m)}$ and $S^{(n-m-1)}$. 
proved it for curves of the fourth order. Cremona proves it for curves of any order.*

I add a theorem about the tangents of these curves.

The tangents of the curve $\mathrm{S}^{(m)}$ are the linear polars of the points on the conjugate curve $\mathrm{S}^{(n-m-1)}$ with respect to the $(m-1)^{\text {th }}$ polars of the corresponding points on the curve $\mathrm{S}^{(m)}$, and these points are the points of contact. They are further the linear polars of the points of contact with respect to the $(n-m)^{t h}$ polars of the corresponding points on $\mathbf{S}^{(n-m-1)}$.

Cremona gives this also for $m=1$. $\dagger$ I shall prove it for $m=2$, the proof being the same in other cases.

Let $\mathrm{P}_{2}=y_{1}^{2} u_{11}+y_{2}^{2} u_{22}+y_{3}^{2} u_{33}+2 y_{2} y_{3} u_{23}+2 y_{3} y_{1} u_{31}+2 y_{1} y_{2} u_{12}$ be the second polar of the point $(y)$ with respect to the original curve $u$. $\mathbf{S}^{(3)}$ is the discriminant of $\mathrm{P}_{2}$, taking the $x$ 's as variables. Now for $\mathrm{S}^{(2)}=0$ and the corresponding $(x)$ on the conjugate curve $S^{(n-3)}$, we have

and

$$
\frac{\delta \mathrm{P}_{2}}{\delta a} \frac{\delta \mathrm{S}^{(2)}}{\delta y_{1}}=\frac{\delta \mathrm{S}^{(2)}}{\delta a} \frac{\delta \mathrm{P}_{2}}{\delta y_{1}}
$$

$$
\frac{\delta \mathrm{P}_{2}}{\delta y_{1}}=\frac{\delta \mathrm{P}_{1}}{\delta x_{1}}=\frac{\delta \mathrm{P}_{n-2}}{\delta y_{1}}
$$

where $\mathrm{P}_{1}$ denotes the first polar of the point $(y)$, and $\mathrm{P}_{n-2}$ the conic polar of $(x)$. The tangent to $\mathrm{S}^{(2)}$ at the point $(y)$ is therefore

$$
z_{1} \frac{\delta \mathrm{P}_{2}}{\delta y_{1}}+z_{2} \frac{\delta \mathrm{P}_{2}}{\delta y_{2}}+z_{3} \frac{\delta \mathrm{P}_{3}}{\delta y_{3}}=0
$$

Instead of this we may write

or

$$
\begin{gathered}
z_{1} \frac{\delta \mathrm{P}_{1}}{\delta x_{1}}+z_{2} \frac{\delta \mathrm{P}_{1}}{\delta x_{2}}+z_{3} \frac{\delta \mathrm{P}_{1}}{\delta x_{3}}=0, \\
z_{1} \frac{\delta \mathrm{P}_{n-2}}{\delta y_{1}}+z_{2} \frac{\delta \mathrm{P}_{n-2}}{\delta y_{2}}+z_{3} \frac{\delta \mathrm{P}_{n-2}}{\delta y_{3}}=0
\end{gathered}
$$

The first of these equations is the linear polar of $(x)$ with respect to $P_{1}$, the first polar of the point $(y)$. The second equation represents the linear polar of $(y)$ with respect to $\mathrm{P}_{n-2}$, the conic polar of the point $(x)$. This is the theorem.

$\mathbf{S}^{(2)}$ is the locus of the double points $(y)$ of the cubic polars; the conjugate curve $S^{(n-3)}$ is the locus of the corresponding poles $(x)$. The conic polar of $(x)$ on $\mathrm{S}^{(n-3)}$ passes through the double point $(y)$ of the cubic polar of $(x)$, and the linear polar of the point $(y)$ to this conic polar is its tangent. But it is also tangent to $\mathbf{S}^{(2)}$. Hence the conic polars of the points on $S^{(n-8)}$ touch the curve $S^{(2)}$. And generally :The $(n-m)^{t h}$ polars of the points on $\mathbf{S}^{(n-m-1)}$ touch the conjugate curve

* Steiner, 1. o. Clebsch, Crelle's Journ., t. 59, §§ 2, 6. Cremona, 1. c., Art. XX. 120,121

† L. o., Art. XX., $118(a)$; XXI., 127 (a). 
$\mathbf{S}^{(m)}$ at the corresponding points. The $(m+1)^{\text {th }}$ polars of the points on $\mathbf{S}^{(m)}$ touch the conjugate curve $\mathbf{S}^{(n-m-1)}$.

This theorem also is given by Cremona for $m=1$ and $m=n-2 . *$

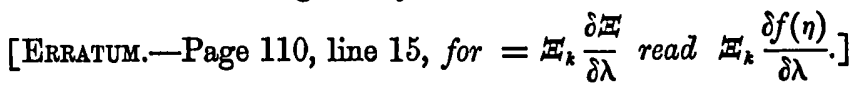

Nov. 26th, 1868.

Prof. SYLVESTER, V.P., in the Chair.

Messrs. R. Abbay, G. H. Darwin, B.A., G. O. Hanlon, and T. Bond Sprague, M.A., were elected Members.

Mr. W. K. Clifford read the following paper:-

On a Generalization of the Theory of Polars.

[The present Note establishes the idea of the polar curve of a curve of given class in respect of a curve of given order, the class being less than the order; and of the polar curve of a curve of given order in respect of a curve of given class, the order being less than the class. It also deals with a certain invariant of two curves, such that the order of one is equal to the class of the other; and with certain other invar riants and contravariants arising out of the theory of polars. I desire to present these ideas by themselves to the Society, because they seem likely to be useful for other purposes than that to which I propose to apply them subsequently, viz., the extension of Grasmann's Geometric Analysis.]

1. Let $B_{n}$ be a curve of the $n$th order, and $c_{m}$ a curve of the $m$ th class. Let the equations of the curres be

$$
\begin{aligned}
& \mathrm{B}_{n} \equiv\left(\mathrm{A}, \mathrm{B}, \mathrm{C}, \mathrm{D} \ldots \ldots . X_{x} y z\right)^{n} \\
& c_{m} \equiv(a, b, c, d \ldots \ldots \ldots \xi \eta \zeta)^{\prime \prime}
\end{aligned}
$$

in point and line coordinates respectively.

In $c_{m}$ write $\frac{\delta}{d x}, \frac{\delta}{d y}, \frac{\delta}{d z}$ in place of $\xi, \eta, \zeta$ respectively, and operate on $B_{n}$ with the symbol thus formed. I denote the result by merely writing $c_{m}$ as an operator before $B_{n}$; thus

then we find

$$
c_{m} \mathrm{~B}_{\mathrm{n}} \equiv\left(a, b, c \ldots . . \chi_{d x}, \frac{\delta}{d y}, \frac{\delta}{d z}\right)^{m} .\left(\mathrm{A}, \mathrm{B}, \mathrm{C}, \ldots \ldots . \chi_{x, y, z)^{n} ;}\right.
$$

(i.) If $m$ is less than $n, c_{m} \mathrm{~B}_{n}$ is a covariant, which $\mathrm{I}$ call the polar curve of $c_{m}$ in respect of $\mathrm{B}_{n}$. It is given in the point coordinates $x, y, z$, and is of order $n-m$.

$$
\text { * L. c., XX., } 118(a) \text {; XXI., } 127 \text { (a). }
$$

